\title{
Cross curvature flow on a negatively curved solid torus
}

\author{
JASON DEBLOIS \\ DAN KNOPF \\ ANDREA YOUNG
}

\begin{abstract}
The classic $2 \pi$-Theorem of Gromov and Thurston constructs a negatively curved metric on certain 3-manifolds obtained by Dehn filling. By Geometrization, any such manifold admits a hyperbolic metric. We outline a program using cross curvature flow to construct a smooth one-parameter family of metrics between the " $2 \pi$-metric" and the hyperbolic metric. We make partial progress in the program, proving long-time existence, preservation of negative sectional curvature, curvature bounds and integral convergence to hyperbolic for the metrics under consideration.
\end{abstract}

$53 \mathrm{C} 44 ;$ 57M50, 58J35, 58J32

\section{Introduction}

In this note, we outline a program that uses cross curvature flow to answer certain questions inspired by the $2 \pi$-Theorem of Gromov and Thurston. We begin and make partial progress toward completing this program. More specifically, we consider cross curvature flow on a class of negatively curved metrics on the solid torus, the simplest nontrivial handlebody. This is motivated by the "Dehn surgery" construction in 3-manifold topology, of which we give a brief account below. We apply the flow to a negatively curved metric described by Gromov-Thurston on a solid torus with prescribed Dirichlet boundary conditions.

Perelman's use of Ricci flow with surgery to prove the Geometrization Conjecture demonstrates the considerable power of geometric flows to address questions about 3-manifolds [25; 26]. Subsequent work, that of Agol-Storm-Thurston [3], for example, shows that Ricci flow may give information even about 3-manifolds known a priori to admit a metric of constant curvature. The results of [3] are obtained by using the monotonicity of volume under Ricci flow with surgery.

Ricci flow is nonetheless an imperfect tool for analyzing hyperbolic 3-manifolds those admitting metrics with constant negative sectional curvatures. According to Geometrization, such manifolds form the largest class of prime 3-manifolds. Paradoxically, they are also the least understood class. In particular, the facts that Ricci flow 
does not preserve negative curvature and that singularities must be resolved by surgery leaves certain basic questions unanswered. For example, one may ask whether the space of negatively curved metrics on a manifold $M$ is connected or contractible, or analogous questions for the space of metrics with negative curvature satisfying various pinching conditions. In higher dimensions, these questions are known to have negative answers (see Farrell and Ontaneda $[14 ; 12 ; 13]$ ); but it has been conjectured and seems plausible that their answers should be positive if $M$ is a hyperbolic 3-manifold.

Such questions have particular relevance to the study of hyperbolic 3-manifolds, because in practice one frequently encounters 3-manifolds on which it is possible explicitly to describe a metric of nonconstant negative curvature and to discern certain geometric information; however, the relationship between this metric and the hyperbolic metric - which exists by Geometrization - is unclear. Such metrics are constructed by Namazi and Souto in [24], for example, as well as in the famous $2 \pi-$ Theorem of Gromov-Thurston [16], which motivates our work here.

The $2 \pi$-Theorem belongs to a family of results describing the geometry of "fillings" that is, manifolds which result from Dehn surgery on cusps of a finite-volume hyperbolic 3 -manifold. We describe this construction in greater detail in Section 2. The initial such result, the "hyperbolic Dehn surgery theorem," of Thurston [28] asserts that most fillings of a hyperbolic 3-manifold admit hyperbolic metrics of their own, but it does not explicitly describe the set of hyperbolic fillings. The $2 \pi$-Theorem remedies this deficiency, but with a weaker conclusion, supplying a metric with negative sectional curvatures on a filling that satisfies a certain explicit criterion. Another result in the spirit of the $2 \pi$-Theorem is the 6-Theorem due to Agol [2] and, independently, Lackenby [21]. This relaxes the hypotheses of the $2 \pi$-Theorem at the expense of weakening its conclusion to a purely topological statement, one that nonetheless implies hyperbolizability using Geometrization.

A program more in the spirit of this paper is that of Hodgson-Kerckhoff [17; 18; 19], which, under somewhat stronger hypotheses, describes a family of "cone" metrics. These are hyperbolic but singular along an embedded one-manifold, and interpolate between the original smooth hyperbolic metric and that on the filling. The goal of our program is to give an alternate construction of an interpolating family. We propose using cross curvature flow (XCF) to construct a one-parameter family of smooth metrics, with negative but nonconstant sectional curvatures, which interpolate between the metric supplied by the $2 \pi$-Theorem and the hyperbolic metric guaranteed by Geometrization.

In [11], Chow and Hamilton introduced the XCF of metrics on a 3-manifold with uniformly signed sectional curvatures. They established certain properties of the flow and conjectured that if the initial datum is a metric with strictly negative sectional 
curvatures, then XCF (suitably normalized) should exist for all time, preserve negative sectional curvature and converge asymptotically to a metric of constant curvature. This conjecture would imply contractibility for the space of negatively curved metrics on a hyperbolic 3-manifold. An additional benefit of a proof of this conjecture is that it should be easier to track the evolution of relevant geometric quantities in a flow without singularities than it is to track their evolution across surgeries.

$\mathrm{XCF}$ is a fully nonlinear, weakly parabolic system of equations, which can be defined as follows: let $P_{a b}=R_{a b}-\frac{1}{2} R g_{a b}$ denote the Einstein tensor, and let $P^{i j}=g^{i a} g^{j b} P_{a b}$. One can define the cross curvature tensor $X$ by

$$
X_{i j}=\frac{1}{2} P^{u v} R_{i u v j}
$$

Then the cross curvature flow of $\left(M^{3}, g_{0}\right)$ is

$$
\begin{aligned}
\frac{\partial}{\partial t} g & =-2 X, \\
g(x, 0) & =g_{0}(x) .
\end{aligned}
$$

Since its introduction in [11], several papers concerning XCF have appeared in the literature. Buckland established short-time existence of XCF for smooth initial data on compact manifolds using DeTurck diffeomorphisms to create a strictly parabolic system [7]. (Like Ricci flow, XCF is only weakly parabolic.) Chen and Ma showed that certain warped product metrics on 2-torus and 2-sphere bundles over the circle are solutions to XCF [22]. Solutions on locally-homogeneous manifolds have recently been studied by Cao, Ni and Saloff-Coste [9; 10]. Glickenstein explored what happens to compact quotients of homogeneous solutions of XCF [15]. The second two authors of this paper have shown that (normalized) XCF is asymptotically stable at hyperbolic metrics [20]. Also, in unpublished earlier work, Andrews has obtained interesting estimates for more general solutions of XCF.

Here we consider a family of negatively curved metrics on the solid torus $D^{2} \times S^{1}$, with initial data that arise in the proof of the $2 \pi$-Theorem. We (1) show short-time existence of XCF with prescribed boundary conditions for metrics in this family, (2) establish curvature bounds depending only on the initial data (showing in particular that negative curvature is preserved for this family), and (3) demonstrate long-time existence.

Although they fall short of confirming the Chow-Hamilton conjectures (even for metrics in this family), our results do provide evidence in favor of those conjectures. On one hand, it is encouraging that negative curvature is preserved and long-time existence holds. On the other hand, the fully nonlinear character of XCF and the high topological complexity of negatively curved manifolds have not yet allowed a complete proof of 
convergence to hyperbolic. In fact, we are not yet able to rule out convergence to a soliton. Note that recent studies of XCF (properly defined) on homogeneous manifolds admitting curvatures of mixed sign display a much greater variety of behaviors than the corresponding examples for Ricci flow (cf $[9 ; 10 ; 15])$.

The purpose of this paper is to introduce our program and to report on partial progress toward its resolution. The paper is organized as follows. In Section 2, we give a brief description of the $2 \pi$-Theorem and related work. In Section 3, we describe the proposed program and give some of its motivations. In Section 4, we derive relevant curvature equations and establish appropriate boundary conditions. In Section 5, we report on our progress thus far, summarizing the new results established in this paper that support the program. To streamline the exposition, the (sometimes lengthy) proofs of these theorems are collected in Section 6.

We hope that the results in this paper contribute to further progress in the program, and ultimately to further applications of curvature flows to the study of hyperbolic 3-manifolds.

The first author is partially supported by NSF grant DMS-0703749. The second author acknowledges NSF support in the form of grants DMS-0505920 and DMS-0545984.

\section{Dehn filling and the $2 \pi$-Theorem}

Suppose $M$ is a compact 3-manifold with a single boundary component: a torus $T^{2} \cong S^{1} \times S^{1}$. A closed manifold may be produced from $M$ by Dehn filling $\partial M$, a well known construction in which a solid torus is glued to $M$ by a homeomorphism of their boundaries. More precisely:

Definition 1 Suppose $M$ is a compact 3-manifold with a torus boundary component $T$. Let $D^{2}$ be the unit disk in $\mathbb{R}^{2}$; let $h$ be a homeomorphism from $\partial\left(D^{2} \times S^{1}\right)=$ $\partial D^{2} \times S^{1}$ to $T$; and for $p \in S^{1}$, take $\lambda=h\left(\partial D^{2} \times\{p\}\right)$. Define the manifold $M(\lambda)$ obtained by Dehn filling $M$ along $\lambda$ by

$$
M(\lambda)=\frac{M \sqcup\left(D^{2} \times S^{1}\right)}{x \sim h(x), x \in \partial D^{2} \times S^{1}} .
$$

We call the isotopy class of $\lambda$ in $T$ the filling slope, and the image in $M(\lambda)$ of $D^{2} \times S^{1}$ the filling torus.

Clearly, the topology of $M(\lambda)$ may vary depending on the choice of $h$, but it is a standard fact that it is determined up to homeomorphism by the choice of filling slope. 
Now suppose that $M$ is a hyperbolic 3-manifold with finite volume. (That is, $M$ is a smooth manifold equipped with a complete, finite-volume Riemannian metric which has sectional curvatures $\equiv-1$.) It follows from the Margulis Lemma [23] (cf [27, Theorem 12.5.1]) that if $M$ is noncompact, each of its cusps is diffeomorphic to $T^{2} \times \mathbb{R}^{+}$. (Here a "cusp" is a component of the complement of a sufficiently large compact submanifold-with-boundary.) Thus removing the cusps of $M$ yields a compact 3-manifold $\widehat{M}$ whose boundary is a disjoint union of tori.

Suppose for simplicity that $M$ has a single cusp. The hyperbolic Dehn surgery Theorem, due to Thurston [28, Section 5], asserts that for all but finitely many choices of slope $\lambda$ on $\partial \widehat{M}$, the closed manifold $\widehat{M}(\lambda)$ admits a hyperbolic metric. In the past thirty years, an enormous quantity of work has been devoted to explicitly describing the set of "hyperbolic filling slopes", and investigating the relationship between the hyperbolic metrics on $M$ and $\widehat{M}(\lambda)$. This is the context of the $2 \pi-$ Theorem, due to Gromov and Thurston [16] (cf [6, Theorem 9]).

A further consequence of the Margulis Lemma is that a parameterization $\phi: T^{2} \times \mathbb{R}^{+} \rightarrow$ $M$ for the cusp of $M$ may be chosen so that the hyperbolic metric on $M$ pulls back to a warped product metric on $T^{2} \times \mathbb{R}^{+}$which restricts on each level torus $T^{2} \times\{t\}$ to a Euclidean metric. (We will precisely describe this metric below.) We call such a parameterization "good".

Theorem 1 (Gromov-Thurston) Let $M$ be a one-cusped hyperbolic 3-manifold with a good parameterization $\phi: T^{2} \times \mathbb{R}^{+} \rightarrow M$ of the cusp of $M$, and suppose $\lambda$ is a slope on $\partial \widehat{M}$ such that $\phi^{-1}(\lambda)$ has a geodesic representative with length greater than $2 \pi$ in some level torus. Then $\widehat{M}(\lambda)$ admits a Riemannian metric with negative sectional curvatures.

The proof is constructive, yielding a metric on $\widehat{M}(\lambda)$ which on $\widehat{M}$ is isometric to the metric inherited from $M$, and on the filling torus has variable negative curvature. Below we will sketch a proof, describing the metrics under consideration on $D^{2} \times S^{1}$. It will be convenient to use cylindrical coordinates $(\mu, \lambda, r) \in[0,1) \times[0,1) \times[0,1]$, where with $D^{2} \times S^{1}$ naturally embedded in $\mathbb{R}^{2} \times \mathbb{R}^{2}$, we have

$$
\begin{array}{ll}
x_{1}=r \cos (2 \pi \mu), & x_{2}=\cos (2 \pi \lambda), \\
y_{1}=r \sin (2 \pi \mu), & y_{2}=\sin (2 \pi \lambda) .
\end{array}
$$

The core of $D^{2} \times S^{1}$ is the set $\{(0,0)\} \times S^{1}=\{(0, \lambda, 0)\}$, and a meridian disk is of the form $D^{2} \times\{p\}=\left\{\left(\mu, \lambda_{0}, r\right)\right\}$ for fixed $p$ or $\lambda_{0}$. The standard rotations of $D^{2} \times S^{1}$ are of the form $(\mu, \lambda, r) \mapsto\left(\mu+\mu_{0}, \lambda, r\right)$, fixing the core and rotating each meridian disk, or $(\mu, \lambda, r) \mapsto\left(\mu, \lambda+\lambda_{0}, r\right)$. All of the metrics we use will be rotationally 
symmetric, and diagonal in cylindrical coordinates, which implies that they have the form

$$
G(r)=f^{2}(r) d \mu^{2}+g^{2}(r) d \lambda^{2}+h^{2}(r) d r^{2} .
$$

Here the functions $f, g$, and $h$ must satisfy the following regularity conditions at $r=0$ to ensure that they extend smoothly across the core.

Lemma $1 G$ extends to a smooth metric on $D^{2} \times S^{1}$ if and only if $g(r)$ and $h(r)$ extend to smooth even functions on $\mathbb{R}$ with $g(0), h(0)>0$, and $f$ extends to a smooth odd function on $\mathbb{R}$ with $f_{r}(0)=2 \pi h(0)$.

Because this is a standard result in Riemannian geometry, we omit the proof.

In a rotationally-symmetric diagonal metric on $D^{2} \times S^{1}$, it will frequently prove convenient to parameterize by an outward-pointing radial coordinate which measures distance from the core:

$$
s(r)=\int_{0}^{r} h(\rho) \mathrm{d} \rho .
$$

Then $G$ may be written in the form

$$
G(s)=f^{2}(s) d \mu^{2}+g^{2}(s) d \lambda^{2}+d s^{2}, \quad s \in\left[0, s_{0}\right] .
$$

Here $s_{0}$ is the distance from the core to the boundary. The smoothness criteria of the lemma above translate to the requirements that $f$ extends to a smooth odd function of $s$, and $g$ to an even function of $s$, such that $f_{s}(0)=2 \pi$ and $g(0)>0$.

Example 1 There is a standard description of the cusp of a hyperbolic manifold as the quotient of a horoball in hyperbolic space $\mathbb{H}^{3}$ by a group of isometries $\Gamma \simeq \mathbb{Z}^{2}$. We will use the upper half-space model for $\mathbb{H}^{3}$, namely $\left\{(x, y, z) \in \mathbb{R}^{3} \mid z>0\right\}$ with the Riemannian metric $G_{h}=z^{-2}\left(d x^{2}+d y^{2}+d z^{2}\right)$. Then a standard horoball is $\mathcal{H}_{1}=\{(x, y, z) \mid z \geq 1\}$, and the elements of $\Gamma$ are Euclidean translations fixing the $z$ coordinate, each of the form $\phi_{(a, b)}:(x, y, z) \mapsto(x+a, y+b, z)$ for $(a, b) \in \mathbb{R}^{2}$.

Without loss of generality, we may assume that $\Gamma$ is generated by elements of the form $\phi_{(M, 0)}$ and $\phi_{\left(x_{0}, L\right)}$, where $M, L>0$. Then $M$ is the length of a "meridian" geodesic of the torus $T=\partial\left(\mathcal{H}_{1} / \Gamma\right)=\{(x, y, 1)\} / \Gamma$, and $L$ is the length of an arc joining the meridian geodesic to itself, perpendicular to it at both ends. (The final constant $x_{0}$ of the definition is a sort of "twisting parameter" and does not enter into computations using the metrics under consideration here.) 
There is a map from the complement of the core in $D^{2} \times S^{1}$ to $\mathcal{H}_{1} / \Gamma$, given in cylindrical coordinates by

$$
\Psi(\mu, \lambda, r)=\left(M \mu, L \lambda, \frac{1}{r}\right), \quad r \in(0,1] .
$$

Using $\Psi$, the hyperbolic metric pulls back to

$$
\Psi^{*} G_{h}=M^{2} r^{2} d \mu^{2}+L^{2} r^{2} d \lambda^{2}+\frac{d r^{2}}{r^{2}}, \quad r \in(0,1] .
$$

Using an outward-pointing radial coordinate which measures distance to the boundary,

$$
s(r)=-\int_{r}^{1} \frac{1}{\rho} \mathrm{d} \rho=\log r,
$$

the hyperbolic metric takes the form

$$
G_{\text {cusp }}=M^{2} e^{2 s} d \mu^{2}+L^{2} e^{2 s} d \lambda^{2}+d s^{2}, \quad s \in(-\infty, 0] .
$$

Sketch of proof of Theorem 1 One seeks a rotationally symmetric diagonal metric on $D^{2} \times S^{1}$ of the form (2-3), which in addition has negative sectional curvatures. One further requires that in a neighborhood $U$ of $\partial\left(D^{2} \times S^{1}\right), f$ and $g$ take the form

$$
f(s)=M e^{s-s_{0}}, \quad g(s)=L e^{s-s_{0}} .
$$

If this is the case, then the map $h:(\mu, \lambda, s) \mapsto\left(\mu, \lambda, s-s_{0}\right)$ takes $U$ isometrically to a neighborhood of $\partial\left(\mathcal{H}_{1} / \Gamma\right)$ with the metric $G_{\text {cusp }}$. Note also that for any $p \in S^{1}$, $\lambda=h\left(\partial D^{2} \times\{p\}\right)$ is a geodesic on $T$ with length $M$; thus in this case, there is a metric on $\widehat{M}(\lambda)$ yielding the theorem. A computation establishes that the sectional curvatures of $G(s)$ are as follows:

$$
K_{\lambda \mu}=-\frac{f_{s} g_{s}}{f g}, \quad K_{\lambda s}=-\frac{g_{s s}}{g}, \quad K_{\mu s}=-\frac{f_{s s}}{f} .
$$

Since $f$ and $g$ are positive on $\left(0, s_{0}\right]$, and $f_{s}(0)=2 \pi$, it follows that all sectional curvatures are negative if and only if $f_{s}, g_{s}, f_{s s}$, and $g_{s s}$ are positive on $\left(0, s_{0}\right]$. Since the components of $G$ must satisfy (2-4) near the boundary, one has $f_{s}\left(s_{0}\right)=M$. Thus since $f_{s s}>0, M$ must be greater than $f_{s}(0)=2 \pi$. One can show that this necessary condition is also sufficient for there to exist a metric of the form (2-3) satisfying our conditions. (See Bleiler and Hodgson [6, Theorem 9].) 


\section{Description of the program}

We now outline our proposed program. As was discussed above, the $2 \pi-$ Theorem constructs a negatively curved metric on certain 3-manifolds obtained by Dehn filling. By Geometrization, any such manifold admits a hyperbolic metric. Our goal is to use XCF to construct a smooth one-parameter family of metrics between the " $2 \pi-$ metric"and the hyperbolic metric. Motivated by the Hodgson-Kerckhoff constructions $[17 ; 18 ; 19]$, one might expect the program to work for slopes $\lambda$ on $\partial \widehat{M}$ such that $\phi^{-1}(\lambda)$ has a geodesic representative of length $\geq L$, for some $L>2 \pi$. (Because our method depends on a stability result, one does not expect to be able to take $L=2 \pi$.)

The hyperbolic Dehn surgery theorem asserts that the manifolds $\widehat{M}(\lambda)$ "approach $M$ geometrically" in the following precise sense: for fixed $z \in \operatorname{int} \widehat{M}$, and $\epsilon, R>0$, there exists $L>0$ such that if $\phi^{-1}(\lambda)$ has geodesic length greater than $L$, there is an injection $f$ taking $B(z, R) \subset M$ to $\widehat{M}(\lambda)$ such that $d\left(f^{*} \mu_{\lambda}, \mu\right)<\epsilon$. Here $\mu_{\lambda}$ and $\mu$ are the (unique) hyperbolic metrics on $M(\lambda)$ and $M$, respectively, and their distance is measured in the $C^{\infty}$ topology. See Benedetti and Petronio [5, Section E.1] or Canary, Epstein and Green [8, Section I.3] for discussions of geometric convergence, and Benedetti and Petronio [5, Section E.5] and Thurston [28, Chapter 5] for statements of the hyperbolic Dehn surgery theorem.

Since $\widehat{M} \subset M$ is compact, for any fixed $z \in \operatorname{int} \widehat{M}$ there is some $R$ such that $\widehat{M} \subset B(z, R)$. The hyperbolic Dehn surgery theorem thus implies that for any $\epsilon>0$, there exists $L>0$ so that for slopes $\lambda$ with geodesic length greater than $L$, the metrics that $\widehat{M}$ inherits from $M$ and $\widehat{M}(\lambda)$, respectively, are $\epsilon$-close in the $C^{\infty}$ topology. Given such a slope $\lambda$, a metric on $\widehat{M}(\lambda)$ that is $\epsilon$-close to the hyperbolic metric may be produced using a metric on $D^{2} \times S^{1}$ that matches $\left.\mu\right|_{\partial \widehat{M}}$ under the gluing map $h$ and is itself $\epsilon$-close to hyperbolic. We hope to use XCF on $D^{2} \times S^{1}$ to flow to a metric with these properties.

The first step is to take as an initial datum a negatively curved metric $G(s, 0)$ on a solid torus given by Equation (2-3). We evolve this metric by cross curvature flow. Because this is a manifold-with-boundary, we impose time dependent Dirichlet boundary conditions corresponding to a homothetically evolving hyperbolic metric. (Example 2 below motivates this choice.) In this paper, we show that the flow exists for all time and preserves negative sectional curvatures. Inspired by the conjecture of Hamilton and Chow, we conjecture that (after normalization) $G(\cdot, t)$ converges uniformly to a hyperbolic metric in $C^{2, \alpha}$ as $t \rightarrow \infty$, at least on sets compactly contained in the complement of the boundary, ie on $[0,1) \times[0,1) \times\left[0, r_{*}\right]$ for any $r_{*} \in(0,1)$. The results in this paper support this conjecture. 
Now suppose the conjecture is proved. Recall that the metric on $\widehat{M}$ is isometric to the hyperbolic metric inherited from $M$. The Dirichlet boundary conditions on the solid torus are deliberately chosen so that at each time, they match the boundary conditions on $\widehat{M}$, with this metric evolved by a homothety. One can argue, assuming convergence on the boundary, that the second fundamental form of our boundary torus should converge to that of a torus in the truncated hyperbolic cusp of $\widehat{M}$. This suggests that for the second step it should be possible, using a local perturbation that is very small for large $t$, to glue the two components while matching the metrics in $\mathcal{C}^{2+\alpha}$. In turn, this allows one to eventually apply the asymptotic stability of hyperbolic metrics under normalized cross curvature flow (proved by the second two authors [20]) to establish exponential convergence to a metric of constant negative curvature. Note that this step will in general change the metric on the entire (closed) manifold.

A successful completion of this program would exhibit a one-parameter family of negatively curved metrics connecting the " $2 \pi$ metric"on $\widehat{M}(\lambda)$ to the hyperbolic metric that, according to Geometrization, this manifold must admit.

\section{Basic equations and boundary conditions}

Let $M^{3}=D^{2} \times S^{1}$ be the solid torus, a 3-manifold with boundary. As above, we restrict our attention to evolving metrics $G$ on $M^{3}$ which are rotationally symmetric. These are metrics that are diagonal in cylindrical coordinates, and for which the isometry group acts transitively on each torus consisting of the locus of points a fixed distance from the core. We may write $G$ as

$$
\begin{aligned}
G(s, t) & =f^{2}(s, t) d \mu^{2}+g^{2}(s, t) d \lambda^{2}+d s^{2} \\
& =e^{2 u(s, t)} d \mu^{2}+e^{2 v(s, t)} d \lambda^{2}+d s^{2}
\end{aligned}
$$

where $s$ is arclength measured outward from the core, as defined in (2-2).

The following example describes the prototypical negatively curved diagonal metric on a solid torus: the hyperbolic metric of constant curvature -1 . This explains our choice of boundary conditions.

Example 2 The metric on a regular neighborhood of a closed geodesic in a hyperbolic manifold is given by

$$
G_{\text {hyp }}=\left(\frac{2}{1-r^{2}}\right)^{2}\left(r^{2} d \mu^{2}+\frac{b}{4}\left(1+r^{2}\right)^{2} d \lambda^{2}+d r^{2}\right)
$$


for $r \in(0,1)$, where $b$ is the length of the geodesic. Using the geodesic radial coordinate

$$
s(r)=\int_{0}^{r} \frac{2}{1-\rho^{2}} \mathrm{~d} \rho=\log \left(\frac{1+r}{1-r}\right),
$$

this takes the form

$$
G_{\text {hyp }}=4 \pi \sinh ^{2} s d \mu^{2}+b \cosh ^{2} s d \lambda^{2}+d s^{2} .
$$

The solution of XCF on any closed hyperbolic manifold with initial data $G_{0}$ is $G(t)=$ $\sqrt{1+4 t} G_{0}$. On the solid torus, the solution of XCF with initial data $G_{\text {hyp }}$ is similarly $G(t)=\sqrt{1+4 t} G_{\text {hyp }}$.

The principal sectional curvatures of the metric $G$ given by (4-1) are

$$
\begin{aligned}
& K_{\lambda \mu}=-\frac{f_{s} g_{s}}{f g}=-u_{s} v_{s}, \\
& K_{\lambda r}=-\frac{g_{s s}}{g}=-\left(v_{s s}+v_{s}^{2}\right), \\
& K_{\mu r}=-\frac{f_{s s}}{f}=-\left(u_{s s}+u_{s}^{2}\right) .
\end{aligned}
$$

We are interested in the case that $G$ has negative sectional curvatures. This occurs when $f$ and $g$, in addition to being positive, are monotonically increasing convex functions of $s$ that satisfy the following conditions at the origin:

$$
\begin{aligned}
& \lim _{s \rightarrow 0} \frac{g_{s}}{f}=\lim _{s \rightarrow 0} \frac{g_{s s}}{f_{s}}=\lim _{s \rightarrow 0} g_{s s}>0, \\
& \lim _{s \rightarrow 0} \frac{f_{s s}}{f}=\lim _{s \rightarrow 0} \frac{f_{s s s}}{f_{s}}=\lim _{s \rightarrow 0} f_{s s s}>0 .
\end{aligned}
$$

One finds from the first equality in (4-3) that the sectional curvatures $K_{\mu \lambda}$ and $K_{r \lambda}$ are identical at the core. This reflects the fact that rotation about the core is an isometry, and so the sectional curvatures tangent to any two planes containing $\partial / \partial \lambda$ are equal.

Henceforth, we assume the initial metric $G_{0}$ has negative sectional curvatures. We denote the absolute values of the principal sectional curvatures by $\alpha, \beta$ and $\gamma$, namely

$$
\alpha=-K_{\lambda \mu}, \quad \beta=-K_{\lambda r}, \quad \gamma=-K_{\mu r} .
$$

The assumption of negative sectional curvature imposes certain constraints on the initial values of $f, g$ and $h$, as mentioned above. Namely, we require that $f(1,0)=\ell_{1}>2 \pi$ and that the initial radius $r_{0}=s(1,0)$ satisfies $1<r_{0}<\ell_{1} / 2 \pi$. We also assume that the metric $G_{0}=G(\cdot, 0)$ obeys a global $C^{2+\theta}$ bound and that $G_{0}$ has constant negative sectional curvature at the core - namely, that $\alpha=\beta=\gamma>0$ at $r=0$. 
We now apply cross curvature flow to a metric of the form (4-1). The evolution of this metric under XCF is equivalent to the system

$$
\begin{aligned}
& f_{t}=\frac{f_{s} g_{s}}{f g} f_{s s}, \\
& g_{t}=\frac{f_{s} g_{s}}{f g} g_{s s}, \\
& h_{t}=\frac{f_{s s} g_{s s}}{f g} h .
\end{aligned}
$$

Notice that these equations remain nondegenerate only as long as $f_{s} g_{s} /(g f)$ remains strictly positive.

Sometimes, it is more tractable to work with the equations for

$$
u=\log f, \quad v=\log g, \quad w=\log h .
$$

These quantities evolve by

$$
\begin{aligned}
u_{t} & =\alpha \gamma=u_{s} v_{s} u_{s s}+u_{s}^{3} v_{s}, \\
v_{t} & =\alpha \beta=u_{s} v_{s} v_{s s}+v_{s}^{3} u_{s}, \\
w_{t} & =\beta \gamma=u_{s s} v_{s s}+v_{s}^{2} u_{s s}+u_{s}^{2} v_{s s}+u_{s}^{2} v_{s}^{2} .
\end{aligned}
$$

Remark 1 Given $w(\cdot, 0)$, the evolution of $w$ is determined by $u_{s}, v_{s}, u_{s s}$, and $v_{s s}$. So we may (and usually do) suppress its equation in what follows.

Remark 2 Observe that system (4-5) for $f$ and $g$ - equivalently, system (4-7) for $u$ and $v-$ is strictly parabolic. This is because our choice to parameterize by arclength $s$ fixes a gauge and thereby breaks the diffeomorphism invariance of XCF. This effectively replaces the DeTurck diffeomorphisms in our proof of short-time existence (Theorem 2, below). This simplification is possible because, in contrast to the more general situation considered by Buckland [7], the spatial dependence of our metrics is only on the radial coordinate $r \in[0,1]$. A similar situation occurs for rotationally invariant Ricci flow solutions; compare the system for $\varphi$ and $\psi$ considered by Angenent and the second author [4], for example. As in that paper, one pays for parabolicity with a commutator; in our case, this is

$$
\left[\partial_{t}, \partial_{s}\right]=-\beta \gamma \partial_{s}
$$


Because the solid torus is a manifold-with-boundary, one must prescribe boundary conditions. We prescribe the Dirichlet boundary conditions given by

$$
\begin{aligned}
u(x, t) & =\log \left(f(1,0)(1+4 t)^{1 / 4}\right), \\
v(x, t) & =\log \left(g(1,0)(1+4 t)^{1 / 4}\right), \\
w(x, t) & =\log \left((1+4 t)^{1 / 4}\right) .
\end{aligned}
$$

As noted above, our choice of boundary conditions are those attained by a homothetically evolving hyperbolic metric.

Lemma 2 As long as a solution exists, the values of the curvatures on the boundary are given by

$$
\alpha(s(1, t), t)=\beta(s(1, t), t)=\gamma(s(1, t), t)=\frac{1}{\sqrt{4 t+1}} .
$$

Proof We define $U(t)=u_{t}(s(1, t), t), V(t)=v_{t}(s(1, t), t)$ and $W(t)=w_{t}(s(1, t), t)$. The result follows from the observation that the evolution equations for $u, v$, and $w$ imply that $\alpha^{2}=U V / W, \beta^{2}=V W / U$, and $\gamma^{2}=U W / V$ on the boundary.

In particular, a simple computation using $G_{\text {hyp }}$ in Example 2 shows that the curvatures on the boundary are those of a homothetically evolving hyperbolic metric.

\section{Summary of results}

We now state our results that establish long-time existence and preservation of negative sectional curvature for the metrics under consideration in this paper. To streamline the exposition, we postpone the proofs until the next section.

\subsection{Long-time existence and uniqueness}

We first establish short-time existence and uniqueness. Since we are considering XCF on a manifold-with-boundary, these results do not immediately follow from [7]. So we use the machinery of [1]. Fix $\theta \in(0,1)$.

Definition 2 Given $\tau>0$, let $E_{\tau}$ denote the space

$$
E_{\tau}:=C^{1+\theta}\left([0, \tau], C^{0}\left(\bar{\Omega}, C^{N}\right)\right) \cap L^{\infty}\left([0, \tau), C^{2+2 \theta}\left(\bar{\Omega}, C^{N}\right)\right),
$$

endowed with the norm

$$
\|\vec{X}\|_{E_{\tau}}:=\sup _{t \in[0, \tau]}\|\vec{X}\|_{C^{0}}+\left[D_{t} \vec{X}\right]_{C^{\theta}}+\sup _{t \in[0, \tau]}\left[D_{x}^{2} \vec{X}\right]_{C^{2 \theta}} .
$$

Here, $C^{r+\theta}$ denotes the usual Hölder norm, and $L^{\infty}$ has its standard meaning. 
Theorem 2 There exists $\tau_{0}>0$ such that the fully nonlinear parabolic system (4-7) has a unique solution $(u, v, w) \in E_{\tau_{0}}$.

It is not known in general whether solutions to cross curvature flow exist for all time. However, in the particular case of our rotationally symmetric metric on the solid torus, we can show a priori bounds on the derivatives of the sectional curvatures.

Theorem 3 Let $G$ be a rotationally symmetric metric on the solid torus given by Equation (4-1). For every $\tau>0$ and $m \in \mathbb{N}$, there exists a constant $C_{m}$ depending on $m, \tau$, and $K$ such that if

$$
\begin{aligned}
& \beta(s, t), \gamma(s, t) \leq K \text { for all } s \in M \text { and } t \in[0, \tau], \\
& \left|\frac{\partial^{m}}{\partial s^{m}} \beta(s, t)\right| \leq \frac{C_{m}}{t^{m}} \text { for all } s \in M \text { and } t \in[0, \tau] \\
& \left|\frac{\partial^{m}}{\partial s^{m}} \gamma(s, t)\right| \leq \frac{C_{m}}{t^{m}} \text { for all } s \in M \text { and } t \in[0, \tau] .
\end{aligned}
$$

then

and

We use these estimates together with the fact that the curvatures stay bounded to show, in the usual way, long-time existence of solutions to Equations (4-5).

Theorem 4 The solution $(u, v, w)$ to XCF on the solid torus exists for all time.

\subsection{Curvature estimates}

One can compute the following evolution equations for the sectional curvatures:

$$
\begin{aligned}
& \alpha_{t}=\alpha \alpha_{s s}+\left(2 \alpha\left(u_{s}+v_{s}\right)+\beta u_{s}+\gamma v_{s}\right) \alpha_{s}+2 \alpha\left(\alpha^{2}-2 \beta \gamma\right), \\
& \beta_{t}=\alpha \beta_{s s}+\left(3 \beta u_{s}+\gamma v_{s}-2 \alpha u_{s}\right) \beta_{s}+2 \beta\left(u_{s}^{2}(\alpha-\beta)-\alpha \gamma\right), \\
& \gamma_{t}=\alpha \gamma_{s s}+\left(3 \gamma v_{s}+\beta u_{s}-2 \alpha v_{s}\right) \gamma_{s}+2 \gamma\left(v_{s}^{2}(\alpha-\gamma)-\alpha \beta\right) .
\end{aligned}
$$

This system is well behaved as long as $\alpha>0$, since this makes $\alpha\left(\partial^{2} / \partial s^{2}\right)$ an elliptic operator. In particular, a maximum principle then applies at interior points. We say $s(r, t)$ is an interior point if $r \in(0,1)$.

Our goal, in the context of Section 3, is to use these evolution equations to show convergence to hyperbolic. Here we collect various estimates that represent progress toward that goal. Define

$$
K_{0}=\sup _{M \times\{0\}}\{\alpha, \beta, \gamma\} \quad \text { and } \quad L_{0}=\inf _{M \times\{0\}}\{\alpha, \beta, \gamma\} .
$$


Theorem 5 (1) For as long as a solution exists, $\alpha>0$. Thus the XCF operator remains elliptic.

(2) The quantities $\alpha, \beta$, and $\gamma$ are bounded from above by $K_{0}$.

(3) The quantities $\alpha, \beta$, and $\gamma$ are bounded from below by $L_{0} e^{-4 K_{0}^{2} t}$.

(4) Negative sectional curvature is preserved.

(5) Over the course of the XCF evolution, one has

$$
\alpha \geq \frac{L_{0}}{4 K_{0} L_{0} t+1} .
$$

Finally, we follow [11] to obtain evidence that our solution converges to a hyperbolic metric in an integral sense. We use their notation and define

$$
J=\int_{M}\left(\frac{P}{3}-(\operatorname{det} P)^{1 / 3}\right) \mathrm{d} V,
$$

where $P=g_{i j} P^{i j}$. Notice the integrand is nonnegative (by the arithmetic-geometric mean inequality) and is identically zero if and only if $g_{i j}$ has constant curvature. Hamilton and Chow's theorem does not directly apply to our setting, as we have a manifold with boundary. However, we are able to prove the analogous theorem.

Theorem 6 Under XCF of a rotationally-symmetric metric on the solid torus, one has

$$
\frac{d J}{d t} \leq 0
$$

\section{Collected proofs}

\subsection{Proofs of long-time existence and uniqueness}

We first use the theory of [1] for short-time existence and regularity of solutions to fully nonlinear parabolic systems. Consider the second-order system

$$
\begin{aligned}
\partial_{t} \vec{X} & =F\left(t, x, \vec{X}, D \vec{X}, D^{2} \vec{X}\right) & & \text { for }(t, x) \text { in }[0, T] \times \bar{\Omega}, \\
H(t, x, \vec{X}, D \vec{X}) & =0 & & \text { for }(t, x) \text { in }[0, T] \times \partial \Omega, \\
\vec{X}(0, x) & =\phi(x) & & \text { for } x \text { in } \bar{\Omega}
\end{aligned}
$$

where $T>0, \Omega$ is a bounded, smooth domain of $\mathbb{R}^{n}$, and $F, H$ are smooth $\mathbb{C}^{N}$-valued functions. 
The short-time existence theory requires the following hypotheses:

- Regularity: The boundary of $\Omega$ and the functions $F, H, \phi$ satisfy

$$
\begin{gathered}
\partial \Omega \in C^{2+2 \theta}, \phi \in C^{2+2 \theta}\left(\bar{\Omega}, \mathbb{C}^{N}\right), \\
F \in C^{2}\left(\Gamma, \mathbb{C}^{N}\right), \\
H \in C^{3}\left(\Gamma^{\prime N}\right),(\theta \in(0,1 / 2)),
\end{gathered}
$$

where $\Gamma:=[0, \infty) \times \bar{\Omega} \times \mathbb{C}^{N} \times \mathbb{C}^{n N} \times \mathbb{C}^{n^{2} N}$ and $\Gamma^{\prime}:=[0, \infty) \times \bar{\Omega} \times \mathbb{C}^{N} \times \mathbb{C}^{n N}$.

- Compatibility: The initial datum $\phi$ satisfies

$$
H(0, x, \phi(x), D \phi(x))=0, x \in \partial \Omega .
$$

If these hypotheses are satisfied, one has:

Theorem 7 There exists $\tau_{0}>0$ such that the fully nonlinear parabolic system has a unique solution $\vec{X} \in E_{\tau_{0}}$, where

$$
E_{\tau}:=C^{1+\theta}\left([0, \tau], C^{0}\left(\bar{\Omega}, C^{N}\right)\right) \cap L^{\infty}\left([0, \tau), C^{2+2 \theta}\left(\bar{\Omega}, C^{N}\right)\right),
$$

with the norm

$$
\|\vec{X}\|_{E_{\tau}}:=\sup _{t \in[0, \tau]}\|\vec{X}\|_{C^{0}}+\left[D_{t} \vec{X}\right]_{C^{\theta}}+\sup _{t \in[0, \tau]}\left[D_{x}^{2} \vec{X}\right]_{C^{2 \theta}} .
$$

Proof of Theorem 2 As explained in Remark 2, the parameterization by arclength $s$, as defined in (2-2), makes XCF a strictly parabolic system; furthermore, this parameterization respects the stated Dirichlet boundary conditions.

Thus we may apply Theorem 7 to the system of equations (4-7) with boundary conditions given by (4-9). Clearly, the regularity hypotheses for $F$ and $H$ are satisfied, because our initial data are $C^{\infty}$. Moreover, one has $u(x, 0)=\log f(1,0), v(x, 0)=\log g(1,0)$, and $w(x, 0)=0$ for $x$ on the boundary torus, so that the compatibility hypothesis is likewise met. Hence Theorem 2 follows.

The proof of long-time existence of solutions to the flow is standard once we obtain a priori derivative estimates. So we will first outline the proof of Theorem 3. We prove this using estimates of Bernstein-Bando-Shi type.

Sketch of proof of Theorem 3 Define $M_{+}:=t \beta_{s}+\lambda_{1} \beta^{2}+\lambda_{2} \gamma^{2}$, where $\lambda_{1}$ and $\lambda_{2}$ are two constants to be chosen later. (We will see that they depend only on the 
initial conditions and the length of the time interval under consideration.) When one computes the evolution of $M_{+}$, one sees that it has the following structure:

$$
\begin{aligned}
\frac{\partial M_{+}}{\partial t}=\alpha\left(M_{+}\right)_{s s}+\left(M_{+}\right)_{s} F_{1}+3 t\left(\beta_{s}\right)^{2} u_{s}+t \beta_{s} \gamma_{s} v_{s} & \\
& +t \beta_{s} F_{2}+t \gamma_{s} F_{3}-2 \alpha \lambda_{1}\left(\beta_{s}\right)^{2}-2 \alpha \lambda_{2}\left(\gamma_{s}\right)^{2},
\end{aligned}
$$

where $F_{i}=F_{i}(\alpha, \beta, \gamma)$ are polynomials depending on the curvatures.

Now let $\tau>0$ be some real number, and consider the evolution equation on $[0, \tau]$. Using bounds on the curvatures and multiple applications of Cauchy-Schwarz, one can show that

$$
\frac{\partial M_{+}}{\partial t} \leq \alpha\left(M_{+}\right)_{s s}+\left(M_{+}\right)_{s} F_{1}+\left(\beta_{s}\right)^{2}\left(F_{2}-2 K \lambda_{1}\right)+\left(\gamma_{s}\right)^{2}\left(F_{3}-2 K \lambda_{2}\right)+F_{4},
$$

where $K>0$ is the lower bound for $\alpha$ on $[0, \tau]$, and the $F_{i}$ (which may differ from above) also depend on $\tau$.

Since all of the $F_{i}$ are bounded from above, we choose $\lambda_{1}$ and $\lambda_{2}$ so that the terms $F_{2}-2 K \lambda_{1}$ and $F_{3}-2 K \lambda_{2}$ are both negative. Then we have

$$
\left(M_{+}\right)_{t} \leq \alpha\left(M_{+}\right)_{s s}+\left(M_{+}\right)_{s} F_{1}+C,
$$

where $C$ is a constant depending only on the initial data. Thus by the parabolic maximum principle, we have

$$
\sup _{x \in M^{3}} M_{+}(x, t) \leq C t+D \leq C \tau+D,
$$

for all $t \in[0, \tau]$, where again $D$ is a constant just depending on the initial data. Hence, on this bounded time interval,

$$
\beta_{s} \leq \frac{C}{t}
$$

Notice that in an analogous fashion, we can obtain a lower bound for $\beta_{s}$ on a bounded time interval by considering $M_{-}:=-t \beta_{s}-\lambda_{1} \beta^{2}-\lambda_{2} \gamma^{2}$. Thus we obtain our desired result that $\left|\beta_{s}\right| \leq C / t$ on $[0, \tau]$, where $C$ depends only on the initial data and on $\tau$.

Similarly, to estimate $\gamma_{s}$, one considers $N_{+}:=t \gamma_{s}+\lambda_{1} \beta^{2}+\gamma^{2}$ along with $N_{-}:=$ $-t \gamma_{s}-\lambda_{1} \beta^{2}-\lambda_{2} \gamma^{2}$ and shows that in fact $\left|\gamma_{s}\right| \leq C / t$, for $C$ as above.

We then use induction to show that higher-order estimates $\left|\partial^{m} \beta / \partial s^{m}\right|,\left|\partial^{m} \gamma / \partial s^{m}\right| \leq$ $C / t^{m}$ hold on $[0, \tau]$ for any $m>0$.

In order to show long-time existence of solutions to the system given by (4-5), we will prove a theorem analogous to that for Ricci flow. 
Theorem 8 Let $G_{0}$ be a metric on the solid torus $M^{3}$ given by (4-1). Then unnormalized cross curvature flow has a unique solution $G(t)$ such that $G(0)=G_{0}$. This solution exists on a maximal time interval $[0, T)$. If $T \leq \infty$, then at least one of

$$
\lim _{t \rightarrow T}\left(\sup _{s \in M^{3}}|\alpha(s, t)|\right), \quad \lim _{t \rightarrow T}\left(\sup _{s \in M^{3}}|\beta(s, t)|\right), \quad \lim _{t \rightarrow T}\left(\sup _{s \in M^{3}}|\gamma(s, t)|\right)
$$

is infinite.

Proof As for Ricci flow, we prove the contrapositive of this statement. Suppose that the solution exists on a maximal time interval $[0, T)$, and that there exists $K>0$ such that $\sup _{0 \leq t<T}|\alpha(s, t)|, \sup _{0 \leq t<T}|\beta(s, t)|$, and $\sup _{0 \leq t<T}|\gamma(s, t)| \leq K$. The key idea is to show that $G(s, T)$ is a smooth limit metric on the solid torus of the form given in (4-1). We can define $f(T)$ and $g(T)$ to be

$$
\begin{aligned}
& f(s, T)=f(s, \tau)+\int_{\tau}^{T} \alpha \gamma f(s, t) \mathrm{d} t, \\
& g(s, T)=g(s, \tau)+\int_{\tau}^{T} \alpha \beta g(s, t) \mathrm{d} t,
\end{aligned}
$$

where $\tau \in[0, T)$ is arbitrary. Using this formulation to compute derivatives of $f(T)$ and $g(T)$, one can use Theorem 3 to bound the curvature quantities, thereby showing that both $f(T)$ and $g(T)$ are smooth. It remains to show that the metric $G(T)=$ $f(T)^{2} d \mu^{2}+g(T)^{2} d \lambda^{2}+d s^{2}$ extends to a smooth metric on the solid torus; namely, that $g(T)$ extends to an even function such that $g(0, T)>0$ and that $f(T)$ extends to an odd function with $f_{s}(0, T)=1$. These facts can be seen from the integral formulation above, if one recalls that the curvatures extend to even functions. Then $G(T)$ is a smooth metric on the solid torus, so Theorem 2 implies that a solution exists on $[T, T+\epsilon)$ for some $\epsilon>0$. This contradicts $T$ being maximal.

Corollary 1 (Theorem 4) Let $G_{0}$ be as in (4-1). Then the solution $G(t)$ to cross curvature flow with $G(0)=G_{0}$ exists for all time.

Proof By Parts (2) and (3) of Theorem 5, we obtain uniform bounds for $\alpha, \beta, \gamma$. Thus Theorem 8 implies $T=\infty$, to wit, that the flow exists for all time.

\subsection{Analysis of core conditions}

Recall that one of our requirements on the metric $G$ was that $f=0$ on the core circle. Then the form in which the evolution equations (4-7) are written involves the quantity $u_{s}=f_{s} / f$, which blows up as $s \searrow 0$. Because of this, we treat the core as a special case for the curvature evolution equations. We do this in a standard way using l'Hôpital's rule, beginning with the following lemma. 
Lemma 3 On the solid torus, one has

$$
\lim _{s \rightarrow 0} u_{s}^{2}(\alpha-\beta)=\lim _{s \rightarrow 0} \frac{1}{3}\left(\beta \gamma-\beta^{2}-\beta_{s s}\right) .
$$

Proof Writing the expression $u_{s}(\alpha-\beta)$ in terms of $f$ and $g$ and their derivatives, we obtain

$$
u_{s}^{2}(\alpha-\beta)=\frac{f_{s}^{2}}{f^{2}}\left(\frac{f_{s} g_{s}}{f g}-\frac{g_{s s}}{g}\right)=\frac{f_{s}^{2}}{g}\left(\frac{f_{s} g_{s}-f g_{s} s}{f^{3}}\right) .
$$

The quantity outside the parentheses above has a well-defined limit as $s \rightarrow 0$, and both the numerator and denominator of the fraction inside the parentheses approach 0 as $s \rightarrow 0$. We apply l'Hôpital's rule three times to obtain the following:

$$
\begin{aligned}
\lim _{s \rightarrow 0} u_{s}^{2}(\alpha-\beta) & =\lim _{s \rightarrow 0} \frac{f_{s}}{g}\left(\frac{f_{s s} g_{s}-f g_{s s s}}{3 f^{2}}\right) \\
& =\lim _{s \rightarrow 0} \frac{1}{g}\left(\frac{f_{s s s} g_{s}+f_{s s} g_{s s}-f_{s} g_{s s s}-f g_{s s s s}}{6 f}\right) \\
& =\lim _{s \rightarrow 0} \frac{1}{3}\left(f_{s s s} \frac{g_{s s}}{g}-\frac{g_{s s s s}}{g}\right) .
\end{aligned}
$$

A quick computation of the limit of $\beta_{s s}$ as $s \rightarrow 0$ reveals that

$$
\lim _{s \rightarrow 0} \beta_{s s}=\lim _{s \rightarrow 0} \frac{g_{s s s s}}{g}-\frac{g_{s s}^{2}}{g^{2}},
$$

and this together with the fact that $f_{s s s}$ and $\gamma$ have the same limit as $s \rightarrow 0$ yields the conclusion.

From the result above, the following description of the curvature evolution at the core follows readily.

Lemma 4 At the core, the evolution equations for the curvatures are as follows:

$$
\begin{aligned}
& \alpha_{t}=4 \alpha \alpha_{s s}+2 \alpha^{3}-4 \alpha^{2} \gamma, \\
& \beta_{t}=\frac{4}{3} \alpha \beta_{s s}-\frac{2}{3} \beta^{3}-\frac{4}{3} \beta^{2} \gamma, \\
& \gamma_{t}=2 \alpha \gamma_{s s}-2 \alpha^{2} \gamma .
\end{aligned}
$$

Proof We recall that the curvatures all extend past the origin to smooth even functions of $s$. Using this fact and l'Hôpital's rule, we have

$$
\lim _{s \rightarrow 0} u_{s} \alpha_{s}=\lim _{s \rightarrow 0} \alpha_{s s},
$$


with similar identities holding for $\beta$ and $\gamma$. The lemma follows readily from this fact and Lemma 3, using the fact that $\alpha=\beta$ at the core.

Remark 3 It may be noted that since $\alpha=\beta$ at the core, their evolution equations should be identical. In fact this is true; the missing ingredient is the fact that

$$
\lim _{s \rightarrow 0} \alpha_{s s}=\lim _{s \rightarrow 0} \frac{1}{3}\left(\beta_{s s}+2 \beta \gamma-2 \beta^{2}\right) .
$$

This may be proved by explicitly writing the formula for $\alpha_{s s}$ in terms of $f$ and $g$ and their derivatives, and using l'Hôpital's rule to simplify the limit as $s \rightarrow 0$, as above.

\subsection{Proofs of curvature estimates}

Here we provide the proofs of the curvature estimates, using the maximum principle.

Lemma 5 As long as a smooth XCF solution exists with $\alpha>0$, one has $\beta \geq 0$ and $\gamma \geq 0$.

Proof This is a standard maximum principle argument, slightly tweaked to accommodate the core and boundary conditions. We give the proof for $\beta$; the proof for $\gamma$ is similar. Suppose a smooth solution with $\alpha>0$ exists on the time interval $[0, \tau]$. Let $C_{0}$ be the maximum attained by the function $u_{s}^{2}(\alpha-\beta)-\alpha \gamma$ on this time interval. (Recall that although $u_{s}$ blows up at the origin, $u_{s}^{2}(\alpha-\beta)$ is at least continuous there by Lemma 3.) Define $C_{1}$ to be the maximum achieved by $-2 / 3\left(\beta^{2}+2 \beta \gamma\right)$ at the core on the time interval $[0, \tau]$, and let $C$ be the maximum of $C_{0}$ and $C_{1}$. Define $\phi(s, t)=e^{-C t} \beta(s, t)$. The evolution equation for $\phi$ is

$$
\phi_{t}=\alpha \phi_{s s}+\left(3 \beta u_{s}+\gamma v_{s}-2 \alpha u_{s}\right) \phi_{s}+\phi\left(u_{s}^{2}(\alpha-\beta)-\alpha \gamma-C\right)
$$

on the interior, and at the core it is

$$
\phi_{t}=\frac{4}{3} \alpha \phi_{s s}+\phi\left(-\frac{2}{3}\left(\beta^{2}+2 \beta \gamma\right)-C\right) .
$$

At a local minimum for $\phi$ in the interior or at the core, one has $\phi_{s s} \geq 0$ and $\phi_{s}=0$; and from our definition of $C$, it follows that if $\phi<0$ at such a local minimum, then $\phi_{t} \geq 0$ there.

For $\delta>0$, consider the function $\phi+\delta(t+1)$. Initially this function has all values larger than 0 , since $\beta$ has all values larger than 0 initially. If there is a first time in $(0, \tau]$ that $\phi(s, t)+\delta(t+1)=0$ for some $s$, then the observations above imply that $(\phi+\delta(t+1))_{t}=\phi_{t}+\delta \geq \delta$ there. (Such a minimum cannot occur on the boundary, 
since values of $\beta$ are always positive there.) But since $t$ is the first time that such a minimum occurs, computing the time derivative $(\phi+\delta(t+1))_{t}$ from below shows that this quantity must be less than or equal to 0 , a contradiction. Thus $\phi+\delta(t+1)$ is positive on $[0, \tau]$, and since $\delta>0$ is arbitrary, so is $\phi$. But then so is $\beta$, since $\phi$ is a positive multiple of $\beta$.

Lemma 6 Suppose a solution exists for $0 \leq t \leq T$. Define $K=\sup _{M^{3} \times[0, T]}\{\beta, \gamma\}$. Then for all $t \in[0, T]$, one has

$$
\alpha_{\min }(t) \geq \alpha_{\min }(0) e^{-4 K^{2} t}
$$

Proof Note that $\alpha_{\min }(0) \leq K$, since the curvatures are all equal to -1 at the origin at time $t=0$. For $\delta>0$, define the barrier function $A(t)=\alpha_{\min }(0) e^{-4 K^{2} t}-\delta$. Then $\alpha \geq A+\delta$ at $t=0$. If there is a first time $t \in(0, T]$ such that $\alpha_{\min }(t)=A(t)$, then $\alpha_{\min }(t)$ is attained either at an interior point or the core.

In the former case, one has

$$
\left\{\begin{array}{l}
\alpha_{t} \leq A^{\prime} \\
\alpha \alpha_{s s}=A \alpha_{s s} \geq 0, \\
\alpha_{s}=0
\end{array}\right.
$$

By hypothesis $\beta \leq K$ and $\gamma \leq K$. Hence at $(s, t)$, one has

$$
A^{\prime 2}(A+\delta) \geq \alpha_{t}>-4 \alpha \beta \gamma \geq-4 K^{2} A .
$$

This is evidently impossible.

If the minimum occurs at the core, then appealing to the evolution equation there shows that $\alpha_{t} \geq-4 \alpha^{2} \gamma \geq-2 A^{2} K$. This yields

$$
A^{\prime 2}(A+\delta) \geq \alpha_{t} \geq-2 A^{2} K .
$$

Since $K \geq \alpha_{\min }(0)$ and $K \geq 1$ we have $K>A$ at $t$. Plugging into the above inequality yields

$$
A^{\prime 2}(A+\delta) \geq-4 A^{2} K>-4 K^{2} A .
$$

This is plainly a contradiction. Since $\delta$ was arbitrary and $\alpha$ is controlled on the boundary, the lemma follows.

Corollary 2 (Part (1) of Theorem 5) For as long as a solution exists, $\alpha>0$.

This follows immediately from the lemma, and shows that the XCF operator remains elliptic for as long as the flow exists.

Now let $K_{0}=\sup _{M \times\{0\}}\{\alpha, \beta, \gamma\}$. 
Lemma 7 Suppose a solution exists for $0 \leq t \leq T$. Define

$$
K^{\prime}=\max \left\{K_{0}, \sup _{M \times[0, T]} \alpha\right\} .
$$

Then with $K$ as in Lemma 6, we have $K \leq K^{\prime}$.

Proof We first consider the case of $\gamma$. The key observation is that at an interior maximum $(s, t)$ with $\gamma(s, t) \geq \alpha$, we have

$$
\gamma_{t}(s, t) \leq 2 \beta\left(v_{s}^{2}(\alpha-\gamma)-\alpha \beta\right) \leq-2 \alpha \beta \gamma \leq 0 .
$$

This follows from the evolution equation for $\gamma$ after observing that at such a point, one has $\alpha \gamma_{s s} \leq 0$ and $\gamma_{s}=0$. The corresponding fact at the core follows from the evolution equations in Lemma 4.

Now for $\delta>0$, define $\gamma_{\delta}(s, t)=\gamma(s, t)-\delta(t+1)$. Then $\gamma_{\delta}$ is initially smaller than $K^{\prime}$. If there is a first time $t$ with $\gamma_{\delta}(s, t)=K^{\prime}$, then by the above we have $\left(\gamma_{\delta}\right)_{t} \leq-\delta$ at such a point. But we must have $\left(\gamma_{\delta}\right)_{t} \geq 0$, a contradiction. Since $\delta>0$ was arbitrary and $\gamma$ is controlled on the boundary, the lemma follows.

The proof for $\beta$ is analogous.

Notice that the estimates of Lemma 6 and Lemma 7 do not give a priori bounds (in terms of the initial data) on the curvatures, as they ultimately rely on the upper bound attained by $\alpha$ over the course of the evolution. We now show that an upper bound is in fact the quantity $K_{0}$.

Lemma 8 For as long as the flow exists, $\alpha(s, t) \leq K_{0}$.

Proof Define $\alpha_{\delta}(s, t)=\alpha(s, t)-\delta(t+1)$. Then $\alpha_{\delta}(\cdot, 0)<K_{0}$. Suppose there is a first time $t_{0}$ at which $\alpha_{\delta}$ attains the value $K_{0}$. We claim that the value $K_{0}+\delta\left(t_{0}+1\right)$ attained by $\alpha$ at $\delta_{0}$ is the maximum value it attains on the interval $\left[0, t_{0}\right]$. To see this, suppose there were some $t<t_{0}$ such that $\alpha(s, t)=K_{0}+\delta\left(t_{0}+1\right)$ for some $s$. Then $\alpha_{\delta}(s, t)=K_{0}+\delta\left(t_{0}-t\right)>K_{0}$, a contradiction to our hypothesis that $t_{0}$ is the first time $\alpha_{\delta}$ attains the value $K_{0}$. Thus by Lemma 7, the values of $\beta$ and $\gamma$ are bounded above by $K_{0}+\delta\left(t_{0}+1\right)$ on the interval $\left[0, t_{0}\right]$. In particular (this is the important point), at the maximum of $\alpha$ at time $t_{0}$, we have $\alpha \geq \beta$ and $\alpha \geq \gamma$. Appealing to the evolution equation for $\alpha$ recorded in (5-1), one may obtain a contradiction in the usual way if the quantity $\alpha^{2}-2 \beta \gamma<0$. The difficult case (and the reason we have not given $a$ priori bounds to this point) is when this inequality does not hold. To deal with this, we use the fact that $\alpha=u_{s} v_{s}$ to rewrite the evolution equation for $\alpha$. Recall that 
$\alpha_{s}=\beta u_{s}+\gamma v_{s}-\alpha\left(u_{s}+v_{s}\right)$. Using this, we rewrite the evolution equation for $\alpha$ as follows:

$$
\begin{aligned}
\alpha_{t} & =\alpha \alpha_{s s}+\left(\beta u_{s}+\gamma v_{s}\right) \alpha_{s}+2 \alpha\left(\left(u_{s}+v_{s}\right) \alpha_{s}+\alpha^{2}-2 \beta \gamma\right) \\
& =\alpha \alpha_{s s}+\left(\beta u_{s}+\gamma v_{s}\right) \alpha_{s}+2 \alpha\left(u_{s}^{2}(\beta-\alpha)+v_{s}^{2}(\gamma-\alpha)+\alpha \beta+\alpha \gamma-\alpha^{2}-2 \beta \gamma\right) \\
& =\alpha \alpha_{s s}+\left(\beta u_{s}+\gamma v_{s}\right) \alpha_{s}-2 \alpha\left(u_{s}^{2}(\alpha-\beta)+v_{s}^{2}(\alpha-\gamma)+(\alpha-\beta)(\alpha-\gamma)+\beta \gamma\right) .
\end{aligned}
$$

At the maximum for $\alpha$ at time $t_{0}$, we have already observed that $\beta \leq \alpha$ and $\gamma \leq \alpha$. Therefore the zero-order term of the differential equation (6-1) is nonpositive, and a contradiction is obtained using the maximum principle in the standard way. Since $\delta>0$ was arbitrary, the Lemma is proved if the maximum occurs in the interior. If the maximum occurs at the core, we note since $\alpha=\beta$ there and $\beta \leq K_{0}+\delta\left(t_{0}+1\right)$, this must also be a local maximum for $\beta$. Using the evolution equation for $\beta$ at the core, we find that $\beta_{t}=\alpha_{t} \leq 0$, also contradiction. Hence the lemma is proved.

The following theorem is an immediate corollary of Lemma 7 and Lemma 8.

Theorem 9 (Part (2) of Theorem 5) For as long as the flow exists, $\alpha, \beta$, and $\gamma$ are bounded above by $K_{0}$.

The universal upper bound of Theorem 9 may be used to give a universal lower bound for the sectional curvatures, as in Lemma 6.

Theorem 10 (Part (3) of Theorem 5) Let $L_{0}=\inf _{M \times\{0\}}\{\alpha, \beta, \gamma\}$. For as long as the flow exists, $\alpha, \beta$, and $\gamma$ are bounded below by $L_{0} e^{-4 K_{0}^{2} t}$.

Proof The case of $\alpha$ follows immediately from Lemma 6, after noting that Theorem 9 implies that the constant $K$ in the Lemma is less than or equal to $K_{0}$, and that $\alpha_{\min }(0) \geq L_{0}$.

We next address the case of $\gamma$. As in the proof of Lemma 6 , for $\delta>0$ we define a barrier function $A(t)=L_{0} e^{-4 K_{0}^{2} t}-\delta$. Then $\gamma>A$ at $t=0$. If there is a first time $t>0$ such that $\gamma_{\min }(t)=A(t)$, then at such a point one has

$$
\left\{\begin{array}{l}
\gamma_{t} \leq A^{\prime} \\
\alpha \gamma_{s s} \geq 0 \\
\gamma_{s}=0
\end{array}\right.
$$

Note that at this point, one has $\alpha>\gamma$, since the inequality holds for $\alpha$. If the minimum occurs in the interior, then by the evolution equation satisfied by $\gamma$ there and the fact that $\alpha \leq K_{0}$ and $\beta \leq K_{0}$, we have

$$
A^{\prime}=-4 K_{0}^{2}(A+\delta) \geq \gamma_{t}>-2 \alpha \beta \gamma \geq-2 K_{0}^{2} A \text {. }
$$


This is a contradiction. If the minimum occurs at the core, appealing to the evolution equation there (see Lemma 4) yields

$$
A^{\prime}=-4 K_{0}^{2}(A+\delta) \geq \gamma_{t}>-2 \alpha^{2} \gamma \geq-2 K_{0}^{2} A,
$$

again yielding a contradiction. Since $\delta>0$ is arbitrary the result is proved for $\gamma$. The case of $\beta$ is analogous.

Theorem 9 and Theorem 10 combine to give bounds for the sectional curvatures that hold for all positive times.

Theorem 10 has another important consequence:

Corollary 3 (Part (4) of Theorem 5) For as long as a the solution of XCF exists, negative sectional curvature is preserved.

We can slightly improve the lower bound for $\alpha$.

Lemma 9 The evolution equation for $\alpha$ in the interior may be written in the following forms:

$$
\begin{gathered}
\text { (6-2a) } \alpha_{t}=\alpha \alpha_{s s}+\left(\beta u_{s}+\gamma v_{s}+2 \alpha\left(u_{s}+v_{s}\right)\right) \alpha_{s}+2 \alpha\left(\alpha^{2}-2 \beta \gamma\right) \\
(6-2 \mathrm{~b}) \quad \alpha_{t}=\alpha \alpha_{s s}+\left(\gamma v_{s}-3 \beta u_{s}+2 \alpha\left(u_{s}+v_{s}\right)\right) \alpha_{s}+4 u_{s}^{2} \beta(\beta-\alpha)-2 \alpha^{2}(2 \beta-\alpha) \\
(6-2 \mathrm{c}) \quad \alpha_{t}=\alpha \alpha_{s s}+\left(\beta u_{s}-3 \gamma v_{s}+2 \alpha\left(u_{s}+v_{s}\right)\right) \alpha_{s}+4 v_{s}^{2} \gamma(\gamma-\alpha)-2 \alpha^{2}(2 \gamma-\alpha) \\
(6-2 \mathrm{~d}) \quad \alpha_{t}=\alpha \alpha_{s s}+\left(\beta u_{s}+\gamma v_{s}\right) \alpha_{s} \\
-2 \alpha\left(u_{s}^{2}(\alpha-\beta)+v_{s}^{2}(\alpha-\gamma)+(\alpha-\beta)(\alpha-\gamma)+\beta \gamma\right)
\end{gathered}
$$

Proof Equations (a) and (d) above were previously obtained; they are Equations (5-1a) and (6-1), respectively. Equation (b) is obtained from (a) by separating off a factor of $4 \beta u_{s} \alpha_{s}$, so that one obtains

$$
\alpha_{t}=\alpha \alpha_{s s}+\left(\gamma v_{s}-3 \beta u_{s}+2 \alpha\left(u_{s}+v_{s}\right)\right) \alpha_{s}+4 \beta u_{s} \alpha_{s}+2 \alpha^{3}-4 \alpha \beta \gamma .
$$

It is easily computed that $\beta u_{s} \alpha_{s}=\beta u_{s}^{2}(\beta-\alpha)+\alpha \beta \gamma-\alpha^{2} \beta$. Substituting this in the equation above and simplifying yields the result. Equation (c) is obtained analogously, but by separating off a factor of $4 \gamma v_{s} \alpha_{s}$ instead of $4 \beta u_{s} \alpha_{s}$.

We use the new equations below to improve the lower bound on the decay of $\alpha$ from exponential to polynomial. Recall that $K_{0}$ is the supremum of the curvatures of the initial metric, and that $L_{0}$ is the infimum. 
Theorem 11 (Part (5) of Theorem 5) Over the course of the evolution, one has

$$
\alpha \geq \frac{L_{0}}{4 K_{0} L_{0} t+1} .
$$

Proof For $\delta>0$, we define the barrier function

$$
A(t)=\frac{L_{0}}{4 K_{0} L_{0} t+1}-\delta,
$$

and note that $\alpha(\cdot, 0)>A(0)$. Suppose there is a first time $t>0$ at which $\alpha(s, t)=A(t)$ for some $s$. If this point $s$ is in the interior, the fact that it is a minimum for $\alpha$ implies that $\alpha_{s s} \geq 0$ and $\alpha_{s}=0$ there. Furthermore, we must have

$$
\alpha_{t} \leq A^{\prime}(t)=-4 K_{0}(A+\delta)^{2} .
$$

At this point the analysis breaks up into three cases depending on the relationship of $\alpha$ with the other curvatures.

Suppose first that $\alpha(s, t)<\beta(s, t)$. Then using Equation (6-2a), we see from the above that

$$
-4 K_{0}(A+\delta)^{2} \geq \alpha_{t}=4 u_{s}^{2} \beta(\beta-\alpha)-2 \alpha^{2}(2 \beta-\alpha)>-4 K_{0} \alpha^{2}=-4 K_{0} A^{2} .
$$

This is a contradiction.

If $\alpha(s, t)<\gamma(s, t)$, an analogous analysis using Equation (6-2b) yields a contradiction in the same way.

It remains to consider the case that $\alpha \geq \beta, \gamma$. In this case, we use the original evolution equation (5-1) for $\alpha$. This yields

$$
-4 K_{0}(A+\delta)^{2} \geq \alpha_{t}=2 \alpha\left(\alpha^{2}-2 \beta \gamma\right) \geq-2 \alpha^{3} \geq-2 K_{0} A^{2},
$$

and again a contradiction is obtained.

Finally, if the minimum occurs at the core, appealing to the evolution equation there (see Lemma 4) yields

$$
\alpha_{t}=-2 \alpha^{2}(2 \gamma-\alpha) \geq-4 K_{0} \alpha^{2},
$$

and an analysis similar to the above yields a contradiction. Since $\delta>0$ was arbitrary, the result is proved. 


\subsection{Integral convergence to hyperbolic}

Here we prove Theorem 6. Recall that we defined

$$
J=\int_{M}\left(\frac{P}{3}-(\operatorname{det} P)^{1 / 3}\right) \mathrm{d} V
$$

in (5-2), where $P$ here denotes the trace of the Einstein tensor. Notice that the integrand is nonnegative (by the arithmetic-geometric mean inequality) and is identically zero if and only if the metric has constant curvature.

The only difficulty is that we are considering a manifold-with-boundary. Because the proof of monotonicity in [11] relies on integration by parts, we must be able to control the boundary terms that arise in our situation. In what follows, we adopt their notation and use the following result.

Lemma 10 (Chow-Hamilton) The evolution of the Einstein tensor under XCF is

$$
\frac{\partial}{\partial t} P^{i j}=\nabla_{k} \nabla_{l}\left(P^{k l} P^{i j}-P^{i k} P^{j l}\right)-\operatorname{det} P g^{i j}-X P^{i j},
$$

where $X=g^{i j} X_{i j}$ is the trace of the cross curvature tensor.

Proof See Chow and Hamilton [11].

Lemma 11 For every smooth $\phi$ defined on our solid torus solution, one has

$$
\frac{d}{d t} \int_{0}^{s_{i}(t)} \phi \mathrm{d} s=\int_{0}^{s_{1}(t)}\left(\phi_{t}+\beta \gamma\right) \mathrm{d} s .
$$

Proof Straightforward computation.

We now prove the final result of this paper.

Proof of Theorem 6 We begin by computing

$$
\begin{aligned}
& \frac{d}{d t} \int_{0}^{s_{1}(t)} P \mathrm{~d} s \\
& =\int_{0}^{s_{1}(t)}\left(P_{t}+\beta \gamma P\right) \mathrm{d} s \\
& =\int_{0}^{s_{1}(t)}\left(g_{i j}\left(\nabla_{k} \nabla_{l}\left(P^{k l} P^{i j}-P^{i k} P^{j l}\right)\right)-3 \operatorname{det} P-X P+2 X_{i j} P^{i j}+\beta \gamma P\right) \mathrm{d} s \\
& =\int_{0}^{s_{1}(t)}(3 \operatorname{det} P-\alpha \beta P-\alpha \gamma P) \mathrm{d} s+\left.\left\langle\frac{\partial}{\partial s}, P^{k l} \nabla_{l} P-P^{j l} \nabla_{l} P_{j}^{k}\right\rangle\right|_{0} ^{s_{1}(t)} .
\end{aligned}
$$


In terms of our metric, the boundary term becomes

$$
\left.\left(\alpha\left(\beta_{s}+\gamma_{s}\right)-\frac{\beta f_{s}(\alpha-\beta)}{f}-\frac{\gamma g_{s}(\alpha-\gamma)}{g}\right)\right|_{0} ^{s_{1}(t)} .
$$

Let $V_{i j}$ denote the inverse of $P^{i j}$. Then

$$
\begin{aligned}
\frac{d}{d t} \int_{0}^{s_{1}(t)}(\operatorname{det} P)^{1 / 3} \mathrm{~d} s & \\
= & \int_{0}^{s_{1}(t)}\left(\partial_{t}(\operatorname{det} P)^{1 / 3}+\beta \gamma(\operatorname{det} P)^{1 / 3}\right) \mathrm{d} s \\
= & \int_{0}^{s_{1}(t)}(\operatorname{det} P)^{1 / 3}\left(\frac{1}{3} V_{i j} \nabla_{k} \nabla_{l}\left(P^{k l} P^{i j}-P^{i k} P^{j l}\right)\right) \mathrm{d} s \\
& \left.\quad+\int_{0}^{s_{1}(t)}(\operatorname{det} P)^{1 / 3}\left(\frac{1}{3} V_{i j}\left(-\operatorname{det} P g^{i j}-X P^{i j}\right)+2 X\right)+\beta \gamma\right) \mathrm{d} s \\
= & : I_{1}+I_{2} .
\end{aligned}
$$

Let us first consider $I_{2}$. Because $V=(\operatorname{det} P)^{-1} X$, one has

$$
\begin{aligned}
\int_{0}^{s_{1}(t)}(\operatorname{det} P)^{1 / 3}\left(\frac{1}{3} V_{i j}\left(-\operatorname{det} P g^{i j}-X P^{i j}\right)\right. & +2 X)+\beta \gamma) \mathrm{d} s \\
& =\int_{0}^{s_{1}(t)}(\operatorname{det} P)^{1 / 3}\left(\frac{1}{3} X-\alpha \gamma-\alpha \beta\right) \mathrm{d} s .
\end{aligned}
$$

Now we do integration by parts on $I_{1}$ to obtain

$$
\begin{gathered}
I_{1}=-\frac{1}{2} \int_{0}^{s_{1}(t)} \nabla_{k}\left((\operatorname{det} P)^{1 / 3} V_{i j}\right)\left(P^{k l} \nabla_{l} P^{i j}-P^{j l} \nabla_{l} P^{i k}\right) \mathrm{d} s \\
+\left\langle(\operatorname{det} P)^{1 / 3} V_{i j} \otimes \frac{\partial}{\partial s}, P^{k l} \nabla_{l} P^{i j}-\left.P^{j l} \nabla_{l} P^{i k}\right|_{0} ^{s_{1}(t)}\right. \\
=\frac{1}{3} \int_{0}^{s_{1}(t)}\left(\frac{1}{2}\left|E^{i j k}-E^{j i k}\right|_{V}^{2}+\frac{1}{6}\left|T^{i}\right|_{V}^{2}\right)(\operatorname{det} P)^{1 / 3} \mathrm{~d} s \\
+\left.\left\langle(\operatorname{det} P)^{1 / 3} V_{i j} \otimes \frac{\partial}{\partial s}, P^{k l} \nabla_{l} P^{i j}-P^{j l} \nabla_{l} P^{i k}\right\rangle\right|_{0} ^{s_{1}(t)},
\end{gathered}
$$

where $E^{i j k}$, et cetera, have the same meanings as in [11]. 
A computation shows that the boundary term is

$$
\begin{aligned}
\left\langle(\operatorname{det} P)^{1 / 3} V_{i j} \otimes \frac{\partial}{\partial s},\right. & \left.P^{k l} \nabla_{l} P^{i j}-P^{j l} \nabla_{l} P^{i k}\right\rangle\left.\right|_{0} ^{s_{1}(t)} \\
& =\left.(\operatorname{det} P)^{1 / 3} V_{i j} T^{1 i j}\right|_{0} ^{s_{1}(t)} \\
& =\left.(\alpha \beta \gamma)^{1 / 3}\left(\frac{\alpha}{\beta} \beta_{s}+\frac{\alpha}{\gamma} \gamma_{s}+\frac{f_{s}}{f}(\beta-\alpha)+\frac{g_{s}}{g}(\gamma-\alpha)\right)\right|_{0} ^{s_{1}(t)} .
\end{aligned}
$$

Now we collect all of the terms above to see that

$$
\begin{aligned}
\frac{d}{d t} J= & \int_{0}^{s_{1}(t)}\left(\operatorname{det} P-\frac{1}{3} \alpha \beta P-\frac{1}{3} \alpha \gamma P-(\operatorname{det} P)^{1 / 3}\left(\frac{X}{3}-\alpha \gamma-\alpha \beta\right)\right) \mathrm{d} s \\
=- & \frac{1}{3} \int_{0}^{s_{1}(t)}\left(\frac{1}{2}\left|E^{i j k}-E^{j i k}\right|_{V}^{2}+\frac{1}{6}\left|T^{i}\right|_{V}^{2}\right)(\operatorname{det} P)^{1 / 3} \mathrm{~d} s \\
& +\alpha\left(\beta_{s}+\gamma_{s}\right)-\frac{\beta f_{s}(\alpha-\beta)}{f}-\left.\frac{\gamma g_{s}(\alpha-\gamma)}{g}\right|_{0} ^{s_{1}(t)} \\
& -\left.(\alpha \beta \gamma)^{1 / 3}\left(\frac{\alpha}{\beta} \beta_{s}+\frac{\alpha}{\gamma} \gamma_{s}+\frac{f_{s}}{f}(\beta-\alpha)+\frac{g_{s}}{g}(\gamma-\alpha)\right)\right|_{0} ^{s_{1}(t)} .
\end{aligned}
$$

Hence $\frac{d}{d t} J \leq-\int_{0}^{s_{1}(t)}(\operatorname{det} P)^{1 / 3}\left(\frac{X}{3}-(\operatorname{det} h)^{1 / 3}\right) \mathrm{d} s$

$$
\begin{aligned}
& -\int_{0}^{s_{1}(t)} \alpha \beta\left(\frac{P}{3}-(\operatorname{det} P)^{\frac{1}{3}}\right) \mathrm{d} s \\
& -\int_{0}^{s_{1}(t)} \alpha \gamma\left(\frac{P}{3}-(\operatorname{det} P)^{\frac{1}{3}}\right) \mathrm{d} s \\
& +\left(\begin{array}{c}
\alpha\left(\beta_{s}+\gamma_{s}\right)-\frac{\beta f_{s}(\alpha-\beta)}{f}-\frac{\gamma g_{s}(\alpha-\gamma)}{g} \\
-(\alpha \beta \gamma)^{1 / 3}\left(\frac{\alpha}{\beta} \beta_{s}+\frac{\alpha}{\gamma} \gamma_{s}+\frac{f_{s}}{f}(\beta-\alpha)+\frac{g_{s}}{g}(\gamma-\alpha)\right.
\end{array}\right)\left(s_{1}\right) \\
& +(\alpha \beta \gamma)^{1 / 3}\left(\begin{array}{c}
\left(\frac{\alpha}{\beta} \beta_{s}+\frac{\alpha}{\gamma} \gamma_{s}+\frac{f_{s}}{f}(\beta-\alpha)+\frac{g_{s}}{g}(\gamma-\alpha)\right. \\
-\alpha\left(\beta_{s}+\gamma_{s}\right)-\frac{\beta f_{s}(\alpha-\beta)}{f}-\frac{\gamma g_{s}(\alpha-\gamma)}{g}
\end{array}\right)(0) .
\end{aligned}
$$

Recall that all of the curvatures are equal at the outer boundary. At the core, $\alpha=\beta$ and all of the curvatures as well as $g$ extend to even functions. Thus all of the boundary terms cancel. The result follows. 


\section{References}

[1] P Acquistapace, B Terreni, Fully nonlinear parabolic systems, from: "Recent advances in nonlinear elliptic and parabolic problems (Nancy, 1988)", (P Bénilan, M Chipot, L C Evans, M Pierre, editors), Pitman Res. Notes Math. Ser. 208, Longman Sci. Tech., Harlow (1989) 97-111 MR1035000

[2] I Agol, Bounds on exceptional Dehn filling, Geom. Topol. 4 (2000) 431-449 MR1799796

[3] I Agol, P A Storm, W P Thurston, Lower bounds on volumes of hyperbolic Haken 3 manifolds, J. Amer. Math. Soc. 20 (2007) 1053-1077 MR2328715 With an appendix by N Dunfield

[4] S Angenent, D Knopf, An example of neckpinching for Ricci flow on $S^{n+1}$, Math. Res. Lett. 11 (2004) 493-518 MR2092903

[5] R Benedetti, C Petronio, Lectures on hyperbolic geometry, Universitext, Springer, Berlin (1992) MR1219310

[6] S A Bleiler, C D Hodgson, Spherical space forms and Dehn filling, Topology 35 (1996) 809-833 MR1396779

[7] J A Buckland, Short-time existence of solutions to the cross curvature flow on 3manifolds, Proc. Amer. Math. Soc. 134 (2006) 1803-1807 MR2207496

[8] R D Canary, D B A Epstein, P Green, Notes on notes of Thurston, from: "Analytical and geometric aspects of hyperbolic space (Coventry/Durham, 1984)", (D B A Epstein, editor), London Math. Soc. Lecture Note Ser. 111, Cambridge Univ. Press (1987) 3-92 MR903850

[9] X Cao, Y Ni, L Saloff-Coste, Cross curvature flow on locally homogenous threemanifolds. I, Pacific J. Math. 236 (2008) 263-281 MR2407107

[10] X Cao, L Saloff-Coste, Cross curvature flow on locally homogenous three-manifolds. II arXiv:0805.3380

[11] B Chow, R S Hamilton, The cross curvature flow of $3{ }^{\circ}$-manifolds with negative sectional curvature, Turkish J. Math. 28 (2004) 1-10 MR2055396

[12] F T Farrell, P Ontaneda, On the moduli space of negatively curved metrics of a hyperbolic manifold arXiv:0805.2635

[13] F T Farrell, P Ontaneda, On the topology of the space of negatively curved metrics arXiv:math/0607367

[14] F T Farrell, P Ontaneda, A caveat on the convergence of the Ricci flow for pinched negatively curved manifolds, Asian J. Math. 9 (2005) 401-406 MR2214959

[15] D Glickenstein, Riemannian groupoids and solitons for three-dimensional homogeneous Ricci and cross-curvature flows, Int. Math. Res. Not. (2008) Art. ID rnn034, 49pp MR2426751 
[16] M Gromov, W Thurston, Pinching constants for hyperbolic manifolds, Invent. Math. 89 (1987) 1-12 MR892185

[17] CD Hodgson, S P Kerckhoff, Rigidity of hyperbolic cone-manifolds and hyperbolic Dehn surgery, J. Differential Geom. 48 (1998) 1-59 MR1622600

[18] C D Hodgson, S P Kerckhoff, Universal bounds for hyperbolic Dehn surgery, Ann. of Math. (2) 162 (2005) 367-421 MR2178964

[19] C D Hodgson, S P Kerckhoff, The shape of hyperbolic Dehn surgery space, Geom. Topol. 12 (2008) 1033-1090 MR2403805

[20] D Knopf, A Young, Asymptotic stability of the cross curvature flow at a hyperbolic metric, Proc. Amer. Math. Soc. 137 (2009) 699-709 MR2448593

[21] M Lackenby, Word hyperbolic Dehn surgery, Invent. Math. 140 (2000) 243-282 MR1756996

[22] L Ma, D Chen, Examples for cross curvature flow on 3-manifolds, Calc. Var. Partial Differential Equations 26 (2006) 227-243 MR2222245

[23] G A Margulis, The isometry of closed manifolds of constant negative curvature with the same fundamental group, Dokl. Akad. Nauk SSSR 192 (1970) 736-737 MR0266103

[24] H Namazi, J Souto, Heegaard splittings and pseudo-Anosov maps, Preprint Available at http://www.math.uchicago.edu/ juan/pseudo8.pdf

[25] G Perelman, The entropy formula for the Ricci flow and its geometric applications arXiv:math.DG/0211159

[26] G Perelman, Ricci flow with surgery on three-manifolds arXiv:math.DG/0303109

[27] J G Ratcliffe, Foundations of hyperbolic manifolds, Graduate Texts in Math. 149, Springer, New York (1994) MR1299730

[28] W P Thurston, The geometry and topology of three-manifolds, Princeton Univ. Math. Dept. Lecture Notes (1979) Available at http://msri.org/publications/books/ gt $3 \mathrm{~m} /$

Department of Mathematics, Statistics and Computer Science, University of Illinois at Chicago 322 Science and Engineering Offices (M/C 249), 851 S Morgan Street, Chicago, IL 60607-7045, USA

Department of Mathematics, University of Texas at Austin

1 University Station C1200, Austin, TX 78712-0257, USA

Department of Mathematics, University of Arizona

617 N Santa Rita Ave, PO Box 210089, Tucson, AZ 85721-0089, USA

jdeblois@math.uic.edu, danknopf@math.utexas.edu, ayoung@math.arizona.edu 
http://www.math.uic.edu/ jdeblois/, http://www.ma.utexas.edu/users/ danknopf, http://math.arizona.edu/ ayoung

Received: 24 June 2009 Revised: 25 November 2009 